\title{
Variables predictoras de la ansiedad precompetitiva: aspectos diferenciales en corredores de fondo en ruta*
}

\author{
Predictors of Precompetitive Anxiety: Differential \\ Aspects in Runners in Route
}

Recibido: 25 de julio de 2014 | Revisado: 28 de julio de 2015 | Aceptado: 28 de julio de 2015

\author{
FRANCISCO RUIZ-JUAN ** \\ Universidad de Murcia, España \\ Antonio ZARAUZ SANCHO **** \\ IES Azcona, Almería, España \\ GABRIEL FLORES-ALLENDE**** \\ Universidad de Guadalajara, México
}

doi:10.11144/Javeriana.upsy14-3.vpap

Para citar este artículo: Ruiz-Juan, F., Zarauz, A., \& Flores-Allende, G. (2015). Variables predictoras de la ansiedad precompetitiva: aspectos diferenciales en corredores de fondo en ruta. Universitas Psychologica, 14(3), 1021-1032. http://dx.doi.org/10.11144/ Javeriana.upsy14-3.vpap

\footnotetext{
* Artículo de investigación

*** Correo electrónico: fruizj@um.es

**** Jefe del Departamento de Educación Física y Deportes del IES Azcona de Almería, España. Contacto principal para la correspondencia. Correo electrónico: tonizarauz@msn.com

****** Correo electrónico: cfdgesa75@hotmail.com
}

\section{RESUMEN}

En la práctica deportiva, estudiar las dimensiones de la ansiedad implicadas en que un sujeto desarrolle dicha actividad se ha convertido en uno de los temas fundamentales de la psicología del deporte y del ejercicio. Se pretendía conocer qué relaciones predictivas presentaban sobre la ansiedad precompetitiva el compromiso a correr, la adicción negativa a correr, las motivaciones y la percepción de éxito en las carreras en ruta. Participaron 1795 corredores de fondo en ruta (1 105 españoles, 690 mexicanos). De ellos, el $85.65 \%$ fueron hombres con una edad de $M=38.98(D E=10.45)$ y el $14.35 \%$ mujeres, con una edad $M=37.88(D E=9.8)$. La recogida de datos se llevó a cabo mediante las adaptaciones al castellano del Revised Competitive State Anxiety Inventory-2 (CSAI-2R), la Commitment to Running Scale-11 (CR-11), la Running Addiction Scale-8 (RAS-8), las escalas Motivations of Marathoners Scales-34 (MOMS-34) y el cuestionario Perception of Success Questionnaire (POSQ). Se obtuvieron valores moderados de ansiedad cognitiva y somática, tanto en hombres y mujeres como en españoles y mexicanos; también elevados valores en autoconfianza, significativamente mayores en hombres respecto a mujeres y en mexicanos respecto a españoles. Con ligeras diferencias por sexo y país, la ansiedad se pudo predecir por puntuar alto en los constructos psicológicos menos deseables analizados (adicción, motivaciones extrínsecas y orientación al ego), mientras que en la autoconfianza fue a la inversa, es decir, por valorar en mayor medida los constructos psicológicos más deseables (compromiso y motivaciones más autodeterminadas).

Palabras clave

ansiedad; compromiso; adicción; motivación; percepción del éxito

\section{A B S T R A C T}

In sports, to study the dimensions of anxiety that involved in individual developing has become one of the key topics of the psychology of sport and exercise. The aim was to find out what had predictive relationships precompetitive anxiety about the commitment to run, run negative addiction, motivations and perceptions of success in road races. Participants 1795 runners en route (1105 Spaniards, Mexicans 690). Of these, $85.65 \%$ were men aged $\mathrm{M}=38.98(\mathrm{SD}=10.45)$ and $14.35 \%$ female, aged $\mathrm{M}=37.88(\mathrm{SD}=$ 9.8). Data collection was conducted by adaptations to the Castilian Revised Competitive State Anxiety Inventory-2 (CSAI-2R), the Commitment to Running Scale-11 (CR-11), the Running Addiction Scale-8 (RAS-8 ) Motivations of Marathoners Scales Scales-34 (MOMS-34) questionnaire 
and Perception of Success Questionnaire (POSQ). Moderate levels of cognitive and somatic anxiety in both men and women and Spaniards and Mexicans were obtained; Also high values in confidence, significantly higher in men versus women and Mexicans with respect to Spanish. With slight differences by sex and country, anxiety could predict score higher in the less desirable psychological constructs analyzed (addiction, extrinsic motivation and guidance to ego), while self-confidence was the reverse, that is, by value in as far as the most desirable psychological constructs (commitment and more self-determined motivation).

Keywords

anxiety; commitment; addiction; motivation; perception of success

\section{Introducción}

La práctica de deportes de manera regular y adecuada al estado físico y edad de cada persona conlleva una larga serie de beneficios para la salud, ya sea a nivel físico, estético o psicológico, que se recogen en multitud de investigaciones; la propia Organización Mundial de la Salud los reconoce y detalla (OMS, 2010), haciendo una serie de recomendaciones al respecto.

Sin embargo, existe un factor que puede condicionar el rendimiento en el deporte, la ansiedad precompetitiva (Anshel, 1995; Gutiérrez, Estévez, García, \& Pérez, 1997; Pozo, 2007; Smith, 1989), puesto que la posibilidad de no conseguir sus objetivos genera en los competidores una incertidumbre que Anshel et al. (1991, p. 9) definieron como: "un sentimiento subjetivo de aprensión o amenaza percibida, a veces acompañada por un incremento de la activación fisiológica".

En la actualidad, en el estudio de la ansiedad precompetitiva se contemplan tres dimensiones: ansiedad cognitiva, ansiedad somática y autoconfianza (Andrade, Lois, \& Arce, 2007; Cox, Martens, \& Russel, 2003). La ansiedad cognitiva evalúa las sensaciones negativas que el sujeto tiene sobre su rendimiento y las consecuencias del resultado. La ansiedad somática hace referencia a la percepción de indicadores fisiológicos de la ansiedad, como tensión muscular, aumento de frecuencia cardíaca, sudoración y malestar en el estómago. La autoconfianza estima el grado de seguridad que el sujeto cree tener acerca de sus posibilidades de éxito en la competición, y no es una medida directa de ansiedad, pero su ausencia es indicador de que el deportista puede experimentar ansiedad cognitiva. Por el contrario, elevados niveles de autoconfianza se asocian positivamente a un mayor rendimiento deportivo (Cantón \& Checa, 2012), especialmente si el nivel de ansiedad cognitiva es moderado o bajo y percibido como facilitador (Mullen, Lane, \& Hanton, 2009).

Los dos estados de ansiedad correlacionan positivamente entre sí y negativamente con la autoconfianza (Buceta, López, Pérez-Llantada, Vallejo, \& Del Pino, 2003; Cervelló, Santos-Rosa, Jiménez, Nerea, \& García, 2002) y, aunque los hombres normalmente muestran mayor autoconfianza que las mujeres (Ruiz-Juan \& Zarauz, 2014; Zarauz \& Ruiz-Juan, 2013a), son ellas quienes controlan mejor su ansiedad (Ponce de León, López, \& Medina, 2006). La edad ha mostrado una relación positiva con la autoconfianza y negativa con la ansiedad somática (Arbinaga \& Caracuel, 2005; Hernández, Olmedilla, \& Ortega, 2008; Zarauz \& Ruiz-Juan, 2013a).

Al margen del sexo y la edad, existen múltiples factores que pueden influir en el grado en que manifiesten los síntomas y dimensiones de la ansiedad precompetitiva, desde el tipo y características del deporte en el que se compite (Jaenes, 2000; Martens, Burton, Vealey, Bump, \& Smith, 1990), al ambiente en el que se desarrolla la competición (Arbinaga \& Caracuel, 2005) y el nivel de la competición (León-Prados, Fuentes, \& Calco, 2011; Pozo, 2007). También pueden influir las características personales de cada deportista, como la experiencia que tenga en competición (Mellalieu, Hanton, \& O’Brien, 2004), la percepción de su grado de preparación para una competición (Gutiérrez et al., 1997) y sus hábitos de entrenamiento (Zarauz \& Ruiz-Juan, 2013b).

De la misma manera, también existen diversos constructos psicológicos que, en función del grado en que se manifiesten en cada deportista, pueden hacer que su ansiedad precompetitiva aumente o disminuya de manera significativa. Así, centrándose en el grado de compromiso con su actividad deportiva, Leedy (2000) concluía que los corredo- 
res de resistencia que mostraban mayor grado de dedicación a su entrenamiento y competición tenían niveles más bajos de ansiedad y depresión que los corredores recreacionales. Sin embargo, Zarauz y Ruiz-Juan (2014) advertían que las dimensiones negativas de la adicción al entrenamiento eran predictoras de la ansiedad cognitiva y somática de los atletas veteranos españoles varones. Además, en esa misma población, Ruiz-Juan, Zarauz y Arbinaga (2013) ya habían obtenido correlaciones positivas entre la adicción al entrenamiento y la ansiedad cognitiva y somática. No obstante, Bueno, Capdevila y Fernández-Castro (2002) concluyeron que los corredores que mostraban mayor autoconfianza antes de la competición, tenían mayores probabilidades de alcanzar sus objetivos. Por el contrario, en los que mostraban mayor ansiedad cognitiva, esas probabilidades eran menores. Respecto a la ansiedad somática, concluyeron que no era demasiado importante, pues desaparecía en gran medida una vez iniciada la competición.

$\mathrm{Al}$ analizar las relaciones de la ansiedad con las motivaciones, centrándose en la teoría de la autodeterminación, Cecchini, González y Contreras (2004), en una muestra de atletas adolescentes, encontraron que la autoconfianza estaba asociada con las motivaciones intrínsecas, mientras que los dos estados de la ansiedad se asociaban con las extrínsecas. A su vez, desde la perspectiva de la teoría de las metas de logro, estos autores concluían que la autoconfianza se asocia a la orientación a la tarea, y los dos estados de la ansiedad, con la orientación al ego de estos jóvenes atletas. Sin embargo, López (2011) halló una alta correlación positiva entre los niveles de ansiedad y motivación extrínseca en porristas de élite, pero ningún resultado significativo en porristas de formación. Recientemente, Zarauz y Ruiz-Juan (2014) concluyeron que en los atletas veteranos varones, la ansiedad cognitiva se podía predecir por puntuar alto en amotivación y orientación al ego, mientras que en las mujeres era la ansiedad somática la que se podía predecir por puntuar alto en amotivación. En las veteranas, además, la autoconfianza se podía predecir por puntuar bajo en motivación intrínseca.
Con respecto a la percepción del éxito en el deporte, Zarauz y Ruiz-Juan (2013c) concluyeron que una mayor ansiedad cognitiva y somática se podía predecir por altos niveles de orientación al ego en los atletas veteranos españoles. Resultados similares obtuvieron Voigh, Callaghan y Ryska (2000) en jóvenes jugadores de voleibol. En jugadores de tenis, Cervelló et al. (2002) obtuvieron que la orientación disposicional al ego se mostraba como predictor significativo y negativo de la ansiedad cognitiva, y la orientación disposicional a la tarea predecía la autoconfianza de forma positiva. Por su parte, Salinero, Ruiz y Sánchez (2006) encontraron que, en karatecas, la orientación a la tarea correlacionaba con la ansiedad. En futbolistas, García-Mas et al. (2011) hallaron una correlación positiva y significativa entre la orientación disposicional al ego y la ansiedad cognitiva; mientras que Olmedilla, Andreu, Ortín y Blas (2009) la obtuvieron negativa y significativa entre la orientación disposicional a la tarea y la ansiedad rasgo. Todos estos datos evidencian que en función del deporte que se practique, la percepción del éxito de sus practicantes influirá de una u otra manera en su ansiedad.

En cualquier caso, en la mayoría de estos estudios, exceptuando algunos realizados con maratonianos y atletas, las muestras empleadas para el análisis de la ansiedad precompetitiva no fueron muy amplias. Por ello, el objetivo de la presente investigación fue analizar los niveles de ansiedad precompetitiva y su predicción por diferentes constructos psicológicos en una extensa muestra de corredores de fondo en ruta. Para ello, se partió de la hipótesis de que en los corredores de ambos sexos existía una moderada ansiedad precompetitiva (cognitiva y somática) y alta autoconfianza. Igualmente, que se iban a obtener unos sólidos modelos predictores de dichos constructos, esperando diferencias entre sexos. Así, con algunas diferencias entre sexos, la ansiedad se podría predecir por puntuar alto en adicción negativa a correr, en las motivaciones menos autodeterminadas y en orientación al ego. Por el contrario, la autoconfianza se podría predecir por puntuar alto en su compromiso a correr, en las motivaciones más autodeterminadas y en orientación a la tarea. 


\section{Método}

\section{Participantes}

Se partió del total de corredores inscritos en los medios maratones de Almería y Elche (España) y Guadalajara (México), en 2010. Para asegurar que la muestra fuera representativa del total de estas carreras (error $\pm 3 \%$, intervalo de confianza $95.5 \%)$, se empleó un diseño de muestreo estratificado por afijación proporcional, teniendo en cuenta el sexo (86.65 \% de hombres y $13.35 \%$ de mujeres) y la edad. Se administró un cuestionario a 1054 corredores de fondo en ruta que participaron en los medios maratones de Almería (30/01/2011), Elche $(03 / 04 / 2011)$ y dos ediciones de Guadalajara (20/02/2011; 19/02/2012).

Además, por medio de una página web se obtuvieron respuestas voluntarias de 741 cuestionarios de corredores de maratón y medio maratón desde 01/03/11 a 20/03/13. Por tanto, uniendo la muestra aleatoria y la voluntaria, se obtuvo una muestra total de 1795 (1 105 españoles, 690 mexicanos) corredores de fondo en ruta, que estaba compuesta por 1541 hombres $(85.65 \%)$ con rango de edad de 18 a 76 años $(M=38.98 ; D E=10.45)$ y 254 mujeres $(14.35 \%)$ con rango de edad de 18 a 69 años $(M=$ $37.88 ;$ DT $=9.8$.

\section{Procedimiento}

En las carreras citadas anteriormente, se pidió permiso a la organización de la carrera mediante una carta en la que se explicaban los objetivos de la investigación, la forma en que se iba a realizar el estudio y se acompañaba un modelo del instrumento. El cuestionario fue administrado en un stand que se dispuso al efecto durante la recogida de dorsales de los atletas participantes el día previo a la carrera.

Para ampliar la muestra y obtener la más amplia y variada geográficamente en el territorio español, se solicitó colaboración al webmaster del principal foro de atletismo de España, al que se le pidió que publicase el cuestionario en la sección de corredores de ruta.
En ambos casos, todos los sujetos fueron informados del objetivo del estudio, de la voluntariedad, absoluta confidencialidad de las respuestas y manejo de los datos, del hecho de que no había respuestas correctas o incorrectas y se les solicitó que contestaran con la máxima sinceridad y honestidad. Además, se pidió que si habían contestado previamente este cuestionario no lo volvieran a hacer. Este trabajo posee el informe favorable de la Comisión de Bioética de la Universidad de Murcia.

\section{Instrumentos}

- Inventario de Ansiedad Competitiva-2 Revisado; versión española de Andrade et al. (2007) de Revised Competitive State Anxiety Inventory-2 (CSAI-2R) de Cox et al. (2003). Tiene 3 subescalas: ansiedad cognitiva, ansiedad somática y autoconfianza. La primera y tercera contienen 5 ítems puntuables de 1 (nada) a 4 (mucho) con lo cual se obtiene una puntuación total entre 5 y 20. La segunda contiene 6 ítems, que ofrece puntuaciones entre 6 y 24.

- Commitment to Running Scale-11 (CR-11) de Ruiz-Juan y Zarauz (2011a); versión española de la Commitment to Running Scale (CR) de Carmack y Martens (1979). Contiene 11 ítems para medir el compromiso a correr (CC) puntuables de 1 (nada de acuerdo) hasta 5 (totalmente de acuerdo), con el que se obtiene una puntuación total entre 11 (mínimo CC) y 55 (máximo CC).

- Running Addiction Scale-8 (RAS-8) de Zarauz y Ruiz-Juan (2011a); versión española de la Running Addiction Scale (RAS) de Chapman y De Castro (1990). Contiene 8 ítems para medir la adicción negativa a correr (ANC) que se puntúan de 1 (nada de acuerdo) hasta 7 (totalmente de acuerdo), con el que se obtiene una puntuación total entre 8 (mínima ANC) y 56 (máxima ANC).

- Motivations of Marathoners Scales-34 (MOMS-34) de Ruiz-Juan y Zarauz (2011b); versión española de la Motivations of Ma- 
rathoners Scales (MOMS) de Masters, Ogles y Jolton (1993). Contiene 7 escalas con 34 ítems que evalúan los motivos para y el grado de orientación motivacional para correr: orientación a la salud, peso, superación de metas personales y competición, reconocimiento, afiliación, meta psicológica y significado de la vida y autoestima. Las respuestas se recogen en una escala tipo Likert desde 1 (no es una razón para correr) hasta 7 (es una razón muy importante para correr), con puntuación total media en cada escala entre 1 (mínima motivación para correr) y 7 (máxima motivación para correr).

- Cuestionario de Percepción de Éxito; versión española de Cervelló (1996) del Perception of Success Questionnaire (POSQ) de Roberts y Balagué (1991). Fue elaborado para medir la orientación de las metas de logro en el contexto deportivo. Consta de 12 ítems, 6 de orientación en la Tarea y 6 en el Ego. La pregunta inicial que encabeza el cuestionario es "Siento éxito en el deporte cuando...." Las respuestas se recogen en escala tipo Likert que oscilan desde totalmente en desacuerdo (1) a totalmente de acuerdo (5).

\section{Análisis de los datos}

Correlación entre las subescalas (coeficiente de Pearson), consistencia interna (alfa de Cronbach), diferencias de medias por países y sexo ( $t$ Student) y regresión lineal multivariante, se realizaron con SPSS 20.0.

\section{Resultados}

\section{Estadística descriptiva y correlación}

Tanto los corredores de fondo en ruta españoles como los mexicanos presentan valores medios en ansiedad precompetitiva (cognitiva y somática), siendo ligeramente superior en los españoles. Sin embargo, los valores en autoconfianza son altos en la muestra española y muy altos en la muestra mexicana. Los hombres en los dos países presentan valores significativamente superiores respecto a las mujeres en autoconfianza, no encontrándose diferencias significativas por sexo en las otras dos variables (Tabla 1).

La Tabla 2 refleja las correlaciones calculadas. Las correlaciones entre la ansiedad cognitiva y la somática son moderadas y positivas, mientras que con la autoconfianza son bajas y negativas, en ambos países y ambos sexos.

\section{Análisis regresivo multivariante}

Se realizó un análisis de regresión lineal multivariado, tratando de obtener unos modelos que explicasen la mayor parte posible de varianza. Se tomaron como variables dependientes las pun-

\section{TABLA 1}

Coeficiente alfa, media, desviación típica, t y significación para ansiedad precompetitiva (CSAI-2R).

Diferencias por sexos y países

\begin{tabular}{|c|c|c|c|c|c|c|c|c|c|c|c|c|c|c|}
\hline & \multicolumn{7}{|c|}{ España } & \multicolumn{7}{|c|}{ México } \\
\hline & \multicolumn{2}{|c|}{$\begin{array}{c}\text { Hombres } \\
n=1007\end{array}$} & \multicolumn{2}{|c|}{$\begin{array}{c}\text { Mujeres } \\
n=98\end{array}$} & \multirow[t]{2}{*}{$t$} & \multirow{2}{*}{$\mathrm{p}$} & \multirow[t]{2}{*}{$\mathrm{d}$} & \multicolumn{2}{|c|}{$\begin{array}{c}\text { Hombres } \\
n=534\end{array}$} & \multicolumn{2}{|c|}{$\begin{array}{c}\text { Mujeres } \\
n=156\end{array}$} & \multirow[t]{2}{*}{$\mathrm{t}$} & \multirow{2}{*}{$\mathrm{p}$} & \multirow[t]{2}{*}{$\mathrm{d}$} \\
\hline & $\alpha$ & $\mathrm{M} \pm \mathrm{DE}$ & $\alpha$ & $\mathrm{M} \pm \mathrm{DE}$ & & & & $\alpha$ & $\mathrm{M} \pm \mathrm{DE}$ & $\alpha$ & $\mathrm{M} \pm \mathrm{DE}$ & & & \\
\hline CSAI-2R & 0.78 & & 0.72 & & & & & 0.78 & & 0.74 & & & & \\
\hline $\begin{array}{l}\text { Ansiedad } \\
\text { cognitiva }\end{array}$ & 0.77 & $1.99 \pm 0.67$ & 0.76 & $2.1 \pm 0.73$ & -1.56 & 0.119 & -0.15 & 0.71 & $1.95 \pm 0.74$ & 0.73 & $1.94 \pm 0.77$ & 0.18 & 0.853 & 0.01 \\
\hline $\begin{array}{l}\text { Ansiedad } \\
\text { somática }\end{array}$ & 0.84 & $2.25 \pm 0.72$ & 0.79 & $2.37 \pm 0.72$ & -1.59 & 0.111 & -0.16 & 0.78 & $2.18 \pm 0.78$ & 0.77 & $2.19 \pm 0.77$ & -0.13 & 0.894 & -0.01 \\
\hline $\begin{array}{l}\text { Autocon- } \\
\text { fianza }\end{array}$ & 0.79 & $3.14 \pm 0.58$ & 0.85 & $3 \pm 0.71$ & 2.2 & 0.028 & 0.21 & 0.72 & $3.56 \pm 0.56$ & 0.74 & $3.45 \pm 0.63$ & 2.06 & 0.039 & 0.18 \\
\hline
\end{tabular}

Fuente: elaboración propia 


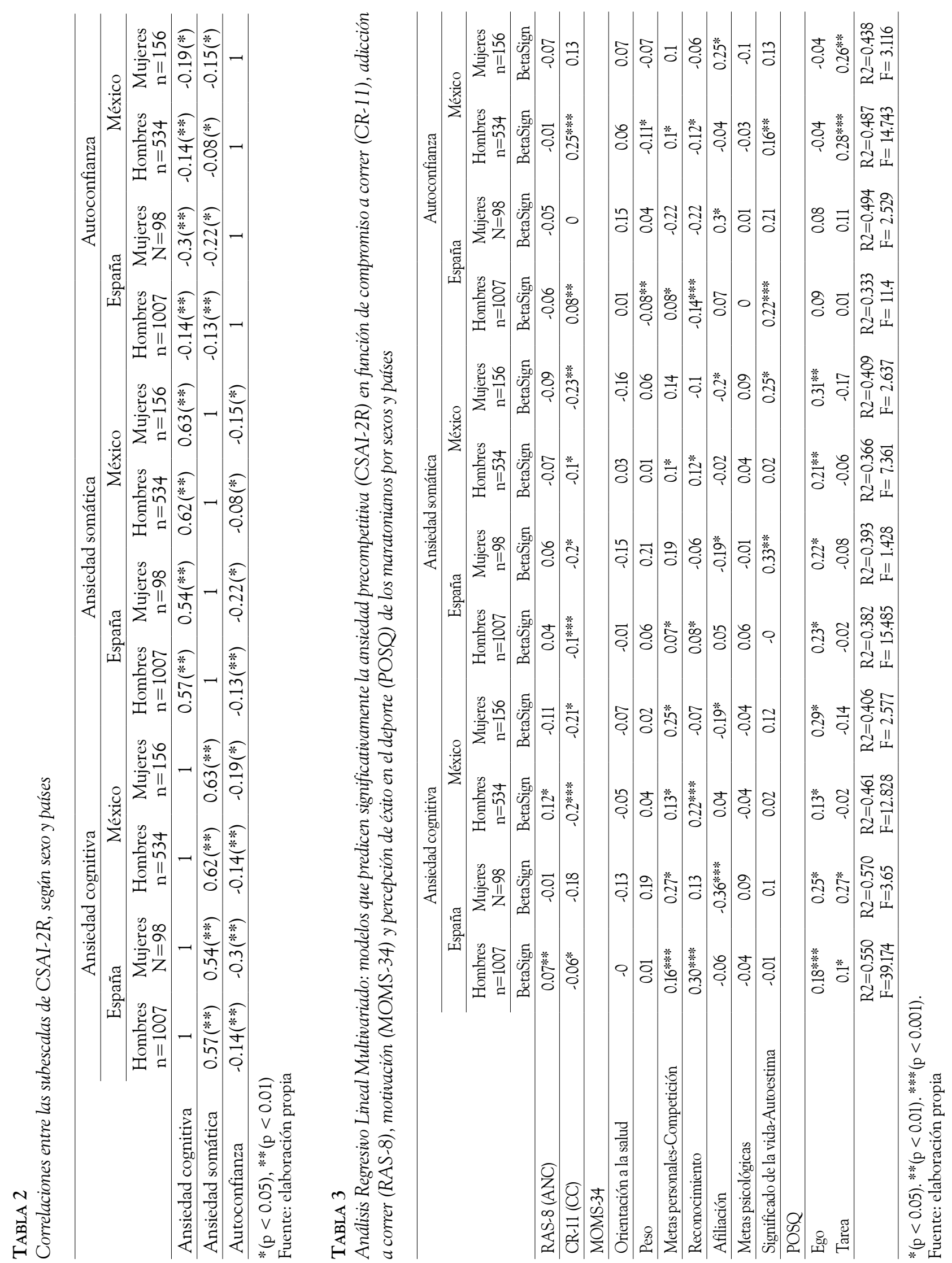


tuaciones medias de la ansiedad precompetitiva (ansiedad cognitiva, ansiedad somática y autoconfianza). Las variables predictoras fueron ANC, CC, cada una de las subescalas de motivación (orientación a la salud, peso, metas personales y competición, reconocimiento, afiliación, metas psicológicas y significado de la vida y autoestima) y percepción de éxito en el deporte (ego y tarea). Como variable de selección se consideró el sexo y el país.

Se extrajo el valor $R^{2}$ para explicar la varianza, Beta para explicar la predicción entre variables, $F$ para ver si existía relación entre variables seleccionadas y su significatividad (Tabla 3). Se obtuvieron modelos sólidos, ya que explicaron entre un tercio y dos tercios de la varianza en hombres y mujeres de ambos países.

El modelo de la ansiedad cognitiva, en hombres de ambos países, fue casi idéntico. Se pudo predecir significativamente por puntuar alto en ANC, metas personales y competición, reconocimiento, ego y tarea (solo en España) y por puntuar bajo en CC (varianza: 55 \% España; 46.1 \% México). También era muy similar el modelo en las mujeres de ambos países, pero con diferencias importantes con los hombres. La predicción fue por puntuar alto en metas personales y competición, ego y tarea (solo en España) y por puntuar bajo en CC y afiliación (varianza: 57 \% España; $40.6 \%$ México).

El modelo de la ansiedad somática, en hombres de ambos países, fue el mismo. Se pudo predecir significativamente por puntuar alto en metas personales-competición, reconocimiento y ego, y por puntuar bajo en CC (varianza: 38.2 \% España; $36.6 \%$ México). El modelo en las mujeres de ambos países también fue idéntico, pero con diferencias importantes con los hombres. La predicción fue por puntuar alto en significado de la vida-autoestima y ego, y por puntuar bajo en CC y afiliación (varianza: 39.3 \% España; 40.9 \% México).

Los hombres españoles y mexicanos presentaron un modelo casi idéntico en autoconfianza ya que esta se pudo predecir por puntuar alto en CC, metas personales-competición, significado de la vida-autoestima y tarea (solo en México) (varianza: 33.3 \% España; 48.7 \% México). El modelo de las mujeres españolas y mexicanas fue casi el mismo y sensiblemente diferente al de los hombres,ya que se predijo por puntuar alto en afiliación y tarea (solo en México) (varianza: 49.4 \% España; 43.8 \% México).

\section{Discusión}

Como se esperaba, los niveles obtenidos en los dos estados de la ansiedad han sido moderados, tanto en hombres y mujeres como en españoles y mexicanos. La explicación puede ser múltiple, puesto que, por un lado, este tipo de pruebas transcurren fuera de un recinto deportivo, normalmente por las calles y parajes más bellos o emblemáticos de una ciudad, lo cual reduce significativamente la ansiedad precompetitiva en los corredores (Jaenes, 2000; Martens et al., 1990). Por otra parte, estos no sienten la presión negativa cercana de miles de espectadores concentrados en un espacio reducido, sino que, por el contrario, se reparten uniformemente a los largo de todo el recorrido para animarlos continuamente, lo que les modera su ansiedad (Arbinaga \& Caracuel, 2005). De la misma manera, con la excepción de las grandes pruebas de carácter internacional y competitivo, estas carreras tienen un carácter meramente popular y participativo, donde la mayoría de atletas corren en grupos de amigos animándose mutuamente, lo que reduce nuevamente la presión que puedan sentir (León-Prados et al., 2011; Pozo, 2007). También, como explicaban Ruiz-Juan y Zarauz (2012), la mayoría de estos atletas tienen muchos años de experiencia entrenando y compitiendo en estas carreras, lo cual también les modera los síntomas de la ansiedad que pudieran padecer (Mellalieu et al., 2004).

En cuanto a los altos valores obtenidos en autoconfianza, al margen de los motivos hasta ahora expuestos que moderan su ansiedad y elevan su autoconfianza, pueden verse incrementados significativamente por los hábitos de entrenamiento de estos deportistas, pues no solo tienen muchos años de experiencia entrenando y compitiendo, sino que además lo hacen durante una media de casi cinco días a la semana (Ruiz-Juan \& Zarauz, 2012), lo cual aumenta su sensación de ir adecuadamente preparados para la competición y, por ello, su autoconfianza 
(Gutiérrez et al., 1997). Además, en estas carreras de larga distancia está prohibida la participación de atletas menores de edad, siendo la mayoría de corredores de edad adulta o madura (Ruiz-Juan \& Zarauz, 2014), lo cual también favorece una mayor autoconfianza (Arbinaga \& Caracuel, 2005; Hernández et al., 2008; Zarauz \& Ruiz-Juan, 2013a).

Las diferencias significativas por sexo obtenidas a favor de los hombres en cuanto a su grado de autoconfianza eran de esperar, puesto que en poblaciones similares de deportistas ya se obtuvieron recientemente (Ruiz-Juan \& Zarauz, 2014; Zarauz \& Ruiz-Juan, 2013a). Sin embargo, que los mexicanos hayan obtenido unos valores significativamente más altos que los españoles en autoconfianza podría ser debido a diferencias culturales y a la manera de entender este deporte en ambas nacionalidades.

Por otra parte, las correlaciones positivas de los dos estados de la ansiedad entre sí y negativas con la autoconfianza, no hacen sino confirmar todo lo hallado en estudios previos (Buceta et al., 2003; Cervelló et al., 2002; Ruiz-Juan et al., 2013), por lo que se tratará de dar explicación por sexo y país de las dimensiones de la ansiedad y autoconfianza a los hallazgos obtenidos en los modelos predictivos.

Así, las bajas puntuaciones obtenidas en su sano y deseable compromiso con su práctica deportiva no solo son predictoras de un mayor nivel en los dos estados de la ansiedad, como ya se obtuviera en diversas investigaciones previas (Leedy, 2000; RuizJuan et al., 2013; Zarauz \& Ruiz-Juan, 2014), sino que las altas puntuaciones en dicho CC predicen también una mayor autoconfianza en los hombres de ambos países. Parece claro que cuanto mayor sea el CC de estos atletas, mayor sensación de ir adecuadamente preparados para la competición tienen y, por ello, mayor autoconfianza, como ya explicaban Gutiérrez et al. (1997).

Sin embargo, considerando la ANC, se obtuvo que unas altas puntuaciones en este constructo son predictoras de la dimensión cognitiva de la ansiedad en los hombres de ambas nacionalidades. Esto es lógico, puesto que los síntomas de la ANC se manifiestan en aquellos corredores que entrenan más días a la semana que en aquellos que simplemente tienen un sano compromiso con su actividad deportiva (Zarauz \& Ruiz-Juan, 2011b), generándose en los corredores adictos una sensación de ir mucho mejor preparados y unas expectativas de éxito mucho mayores que en los comprometidos, lo que hace que se les incremente su ansiedad cognitiva respecto a estos últimos (Gutiérrez et al., 1997).

En cuanto a las motivaciones propias de los corredores de fondo en ruta, se obtuvo que las motivaciones menos autodeterminadas (superación de metas personales-competición y reconocimiento) han sido un claro predictor de las dos dimensiones de la ansiedad en ambos sexos y países, como ya ocurriera en otras poblaciones de deportistas (Cecchini et al., 2004; López, 2011). Además, la más extrínseca de las motivaciones de los corredores varones, el reconocimiento, ha resultado ser también un predictor negativo de la autoconfianza. Esto puede quedar explicado porque los corredores a los que les mueven estas dos motivaciones normalmente son muy adictos, ya que hacen más kilómetros y días de entrenamiento a la semana que el resto (Zarauz \& Ruiz-Juan, 2012), lo cual crea en ellos unas altas expectativas de éxito que, a su vez, son las que les elevan los dos estados de su ansiedad (Gutiérrez et al., 1997).

Considerando ahora las motivaciones más autodeterminadas, la afiliación resultó ser un predictor negativo de la ansiedad cognitiva y somática y positivo de la autoconfianza de todas las corredoras. El hecho de que suceda solo en mujeres podría ser porque los varones normalmente tienen una ANC mucho mayor que las mujeres (Ruiz-Juan \& Zarauz, 2012), por lo que estos se preocupan más por entrenar más días, más horas y más kilómetros a la semana, buscándose un entrenador, pero no amigos con los que correr. Además, Zarauz y RuizJuan (2012) explicaban que a aquellos corredores que les motiva especialmente la afiliación no tienen una elevada ANC ni siquiera un alto compromiso con su práctica deportiva, por lo que su principal objetivo es pasar ratos agradables corriendo en compañía de amigos, de ahí su moderada ansiedad y alta autoconfianza.

Por su parte, la más autodeterminada de las motivaciones de estos corredores, significado de 
la vida-autoestima, ha resultado ser un predictor significativo de la autoconfianza de los varones y de la ansiedad somática de las mujeres, lo cual pone nuevamente de manifiesto las diferencias por sexo ya halladas en estos constructos en esta población (Ruiz-Juan \& Zarauz, 2014). Normalmente, los maratonianos con altas puntuaciones en esta motivación también valoran alto la de reconocimiento, son muy adictos a correr y hacen los hombres más kilómetros que las mujeres (Zarauz \& Ruiz-Juan, 2012). Esto produce en ellos mayor autoconfianza y, en ellas, por la sensación de ir menos preparadas a la competición, mayor ansiedad somática (Gutiérrez et al., 1997).

En cuanto a la percepción del éxito, la orientación al ego de los corredores de ambos países y sexos parece confirmarse como un sólido predictor de los dos estados de la ansiedad, como ocurriera en la mayoría de estudios en otras poblaciones de deportistas (Cervelló et al., 2002; García-Más et al., 2011; Voigh et al., 2000; Zarauz \& Ruiz-Juan, 2013c). Este resultado queda explicado porque, además de la orientación al ego, las motivaciones menos autodeterminadas (reconocimiento y metas personalescompetición) resultaron ser también predictoras de la ansiedad cognitiva y somática. Sin embargo, lo que no se esperaba era que la orientación a la tarea fuese predictora de la ansiedad cognitiva, en España, y en México, de la autoconfianza, lo cual pone nuevamente de relieve las diferencias culturales de entender este deporte en ambas poblaciones.

A modo de conclusiones, parece confirmarse la hipótesis de que, con ligeras diferencias por sexo y país, la ansiedad se puede predecir por puntuar alto en los constructos psicológicos menos deseables analizados (adicción, motivaciones extrínsecas y orientación al ego), mientras que en la autoconfianza es a la inversa, es decir, por valorar en mayor medida los constructos psicológicos más deseables (compromiso y motivaciones más autodeterminadas).

\section{Referencias}

Andrade, E. M., Lois, G., \& Arce, C. (2007). Propiedades psicométricas de la versión española del
Inventario de Ansiedad Competitiva CSAI-2R en deportistas. Psicothema, 19(1), 150-155.

Anshel, M. (1995). Anxiety. En T. Morris \& J. Summers (Eds.), Sport psychology: Theory, applications $\mathcal{E} ?$ issues (pp. 29-62). Brisbane, CA: John Wiley \& Sons.

Anshel, M., Freedson, P., Hamill, J., Haywood, K., Horvat, M., \& Plowman, S. (1991). Dictionary of the sport and exercise sciences. Champaign, IL: Human Kinetics.

Arbinaga, F., \& Caracuel, J.C. (2005). Precompetición y ansiedad en fisicoculturistas. Revista de Psicología del Deporte, 14(2), 195-208.

Buceta, J., López, A., Pérez-Llantada, M., Vallejo, M., \& Del Pino, M. (2003). Estado psicológico de los corredores populares de maratón en los días anteriores a la prueba. Psicothema, 15(2), 273-277.

Bueno, J., Capdevila, L., \& Fernández-Castro, J. (2002). Sufrimiento competitivo y rendimiento en deportes de resistencia. Revista de Psicología del Deporte, 11(2), 209-226.

Cantón, E., \& Checa, I. (2012). Los estados emocionales y su relación con las atribuciones y las expectativas de autoeficacia en el deporte. Revista de Psicología del Deporte, 21(1), 171-176.

Carmack, M. A., \& Martens, R. (1979). Measuring commitment to running: A surrey of runner's attitudes and mental status. Journal of Sport and Exercise Psychology, 1, 25-42.

Cecchini, J. A., González, C., Carmona, A. M., \& Contreras, O. (2004). Relaciones entre clima motivacional, la orientación de meta, la motivación intrínseca, la auto-confianza, la ansiedad y el estado de ánimo en jóvenes deportistas. Psicothema, 16(1), 104-109.

Cervelló, E. (1996). La motivación y el abandono deportivo desde la perspectiva de las metas de logro (Tesis doctoral). Universitat de València, Valencia.

Cervelló, E., Santos-Rosa, F. J., Jiménez, R., Nerea, A., $\&$ García, T. (2002). Motivación y ansiedad en jugadores de tenis. Motricidad, 9, 141-161.

Chapman, C. L., \& De Castro, J. M. (1990). Running addiction: Measurement and associated psychological characteristics. The Journal of Sports Medicine and Physical Fitness, 30(3), 283-290.

Cox, R., Martens, M., \& Russell, W. (2003). Measuring anxiety in athletics: The revised Competitive State 
Anxiety Inventory-2. Journal of Sport and Exercise Psychology, 25(4), 519-533.

García-Más, A., Palou, P., Smith, R. E., Ponseti, X., Almeida, P., Lameiras, ... Leiva, A. (2011). Ansiedad competitiva y clima motivacional en jóvenes futbolistas de competición, en relación con las habilidades y el rendimiento percibido por sus entrenadores. Revista de Psicología del Deporte, 20(1), 197-207.

Gutiérrez, M., Estévez, A., García, J., \& Pérez, H. (1997). Ansiedad y rendimiento atlético en condiciones de estrés: efectos moduladores de la práctica. Revista de Psicología del Deporte, 6(2), 27-46.

Hernández, R., Olmedilla, A., \& Ortega, E. (2008). Ansiedad y autoconfianza de jóvenes judocas en situaciones competitivas de alta presión. Análise Psicológica, 4(26), 689-696.

Jaenes, J. C. (2000). Estado emocional y conducta deportiva: ansiedad competitiva en corredores de maratón (Tesis doctoral inédita). Universidad de Sevilla, España.

Leedy, M. (2000). Commitment to distance running: Coping mechanism or addiction? Journal of Sport Behavior, 23(3), 255- 270.

León-Prados, J., Fuentes, I., \& Calvo, A. (2011). Ansiedad estado y autoconfianza precompetitiva en gimnastas. Revista Internacional de Ciencias del Deporte, 7(23), 76-91.

López, N. I. (2011). Relación entre factores motivacionales y niveles de ansiedad en porristas en precompetencia. International Journal of Psychological Research, 4(1), 80-91.

Martens, R., Burton, D., Vealey, R. S., Bump, L. A., \& Smith, D. E. (1990). The Competitive State Anxiety Inventory-2 (CSAI-2). En R. Martens, R. S. Vealey \& D. Burton (Eds.), Competitive anxiety in sport (pp. 117-213). Champaign IL: Human Kinetics.

Masters, K. S., Ogles B. M., \& Jolton, J. A. (1993). The development of an instrument to measure motivation for marathon running: The Motivations of Marathoners Scales (MOMS). Research Quarterly for Exercise and Sport, 64(2), 134-143.

Mellalieu, S., Hanton, S., \& O’Brien, M. (2004). Intensity and direction of competitive anxiety as a function of sport type and experience. Scandina- vian Journal of Medicine and Science in Sports, 14(5), 326-334. doi: 10.1111/j.1600-0838.2004.00389.x

Mullen, R., Lane, A., \& Hanton, S. (2009). Anxiety symptom interpretation in high-anxious, defensive high-anxious, low-anxious and repressor sport performers. Anxiety, Stress E Coping, 22(1), 91-100. doi: $10.1080 / 10615800802203769$

Olmedilla, A., Andreu, M. D., Ortín, F. J., \& Blas, A. (2009). Ansiedad competitiva, percepción de éxito y lesiones: un estudio en futbolistas. Revista Internacional de Medicina y Ciencias de la Actividad Física y el Deporte, 9(33), 51-66.

Organización Mundial de la Salud. (2010). Recomendaciones mundiales sobre actividad física para la salud. Recuperado el 28 de febrero de 2014 de: http://www.who.int/dietphysicalactivity/factsheet_recommendations/es/

Roberts, G., \& Balagué, G. (septiembre, 1991). The development and validation of the Perception of Success Questionnaire. Paper presented at the FEPSAC Congress, Cologne, Germany.

Ponce de León, Y., López, J., \& Medina, M. (2006). Habilidades psicológicas en los atletas de primera fuerza en atletismo. Revista de Ciencias del Ejercicio - FOD, 2(1), 42-57.

Pozo, A. (2007). Intensidad y dirección de la ansiedad competitiva y expectativas de resultado en atletas y nadadores. Revista de Psicología del Deporte, 16(2), 137-150.

Ruiz-Juan, F., \& Zarauz, A. (2011a). Validación de la versión española de la Commitment to Running Scale (CR). Estudios de Psicología, 32(2), 195-207.

Ruiz-Juan, F., \& Zarauz, A. (2011b). Validación de la versión española de las Motivations of Marathoners Scales (MOMS). Revista Latinoamericana de Psicología, 43(1), 139-156.

Ruiz-Juan, F., \& Zarauz, A. (2012). Variables que hacen adicto negativamente a correr al maratoniano español. Retos, 21, 38-42.

Ruiz-Juan, F., \& Zarauz, A. (2014). Ansiedad en maratonianos en función de variables sociodemográficas. Retos, 25, 28-31.

Ruiz-Juan, F., Zarauz, A., \& Arbinaga, F. (2013). Validación de la Escala de Adicción al Entrenamiento (EAE) en atletas veteranos. Adicciones, 25(4), 309-320. 
Salinero, J. J., Ruiz, G., \& Sánchez, F. (2006). Orientación y clima motivacional, motivación de logro, atribución de éxito y diversión en un deporte individual. Apunts, Educació Física i Esports, 83, 5-11.

Smith, R. (1989). Athletic stress and burnout: Conceptual models and intervention strategies. En D. Hackfort \& C. D. Spielberger (Eds.), Anxiety in sports: An international perspective (pp. 183-201). Nueva York, NY: Hemisphere.

Voight, M., Callaghan, J., \& Ryska, T. (2000). Relationship between goal orientations, selfconfidence and multidimensional trait anxiety among MexicanAmerican female youth athletes. Journal of Sport Behavior, 23(3), 271-288.

Zarauz, A., \& Ruiz-Juan, F. (2011a). Propiedades psicométricas de la versión española de la Running Addiction Scale (RAS). The Spanish Journal of Psychology, 14(2), 967-976.

Zarauz, A., \& Ruiz-Juan, F. (2011b). Compromiso y adicción negativa al entrenamiento y competición de los maratonianos. Revista Internacional de Medicina y Ciencias de la Actividad Física y el Deporte, 11(44), 817-834.

Zarauz, A., \& Ruiz-Juan, F. (2012). Súper-adherencia del maratoniano: variables predictoras y diferencias de género. Universitas Psychologica, 11(3), 895-907.

Zarauz, A., \& Ruiz-Juan, F. (2013a). Análisis de la ansiedad en el atletismo: un estudio con veteranos. Revista Internacional de Ciencias del Deporte, 9(33), 225-235.

Zarauz, A., \& Ruiz-Juan, F. (2013b). Variables predictoras de la ansiedad en atletas veteranos españoles. Retos, 23, 29-32.

Zarauz, A., \& Ruiz-Juan, F. (2013c). Ansiedad, satisfacción, percepción y creencias sobre las causas del éxito en atletas veteranos españoles. Ansiedad y Estrés, 19(1), 83-93.

Zarauz, A., \& Ruiz-Juan, F. (2014). Factores determinantes de la ansiedad en atletas veteranos españoles. Universitas Psychologica, 13(3), 15-25. 
